\title{
Fetal Speckle Tracking Echocardiography: A Comparison Between Fetuses with Normal Heart and Those With Heart Disease
}

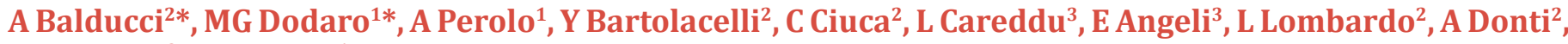 \\ G Gargiulo ${ }^{3}$ and G Pilu ${ }^{1}$
}

${ }^{1}$ Department of Medical and Surgical Science, University of Bologna, Italy

${ }^{2}$ Department of Medical and Surgical Science, University of Bologna, Italy

${ }^{3}$ Department of Pediatric and Adult Congenital Heart Cardiac Surgery, University of Bologna, Italy

*Corresponding author: A Balducci, Department of Medical and Surgical Science, Thoracic and Vascular Sciences, Paediatric Cardiology and Adult Congenital Unit, S. Orsola-Malpighi Hospital, University of Bologna, Bologna, Italy.

Received Date: May 02, 2020

Published Date: May 14, 2020

\begin{abstract}
Objective: The aim of our study was to compare left ventricular function in normal fetal hearts and those with cardiac abnormalities using 2D and 4D e-STIC approach.

Methods: We recruited 63 fetuses between 20- and 40- weeks, including twenty-nine affected by heart disease. All measurements were performed by two sonographers experienced in fetal echocardiography. We compared different parameters obtained by 2-dimensional and 4D e-STIC method in normal fetal hearts and those with cardiac defects. We focused on left ventricular global strain, left ventricular ejection fraction, left ventricular length and area in end-systole and end-diastole, and left ventricular fractional area change. Differences between categorical variables were analyzed by using Chi-Quadro Test, while continuous variables by one-way ANOVA Test.
\end{abstract}

Results: Firstly, no differences were proved between healthy and affected fetuses, regardless of the applied technique. Similarly, fetal strain values did not differ between the affected fetuses distinguished according to the kind of pathology.

Conclusion: In our cohort, 2D and 4D e-STIC imaging techniques have shown similar results for each parameter. We did not find differences between healthy fetuses and those with heart disease because congenital heart disease included did not affect left ventricular systolic function.

Keywords: Echocardiography; Fetal echocardiography; Speckle tracking; Strain; STIC

\section{Introduction}

Echocardiography is the most common tool used to diagnose congenital heart disease. Recently, 2-dimensional speckle tracking echocardiography (STE) has been introduced to investigate cardiac function, identifying the movement of endocardium during the cardiac cycle [1]. STE has been used to study segmental and global cardiac function, calculating velocities and deformation parameters such as longitudinal, radial and circumferential strain and strain rate, which are used to evaluate the myocardial function from an objective and quantitative point of view [2-4]. Moreover, 2D speckle tracking is independent from the heart orientation to the ultrasound beam [5-7]. In addition, thanks to the semiautomated software, it has better intra-observer and inter-observer reproducibility than the other techniques commonly used to evaluate cardiac function [3]. STE is currently applied both in adult and pediatric patients to evaluate ischemic heart disease, left ventricular diastolic dysfunction, myocardial mechanics, cardiomyopathy, vascular disease, and myocardial dysfunction in patients undergoing chemotherapy. Even if it has not yet been validated in the evaluation 
of fetal cardiac dysfunction, it has already been used to measure myocardial deformation in twin-twin transfusion syndrome, fetal cardiomyopathies and structural heart diseases, gestational diabetes, and intrauterine growth restriction [9].

Although there have been differences in findings, STE is considered as an important tool for fetal specialists. However, several issues currently limit its use [8], especially regarding framerate and spatial resolution [9-14]. Recently, three-dimensional (3D) STE has been introduced to overwhelm B-mode imaging limitations. Previous studies proved that four-dimensional (4D) ultrasound technologies, such as spatio-temporal image correlation (STIC), facilitate examination of fetal cardiac images. However, obtaining diagnostic volumes could be limited by fetal movements. Standard mechanical probes allow acquisition of a STIC volume of good quality in 7.5-15 s [15-17]. 4D electronic probes improved image resolution and decreased image artifacts. Electronic STIC acquisition stitches together subvolumes resulting in a higher resolution real time image and in a faster technique $[18,19]$. The aim of our study was to compare left ventricular function in normal fetal hearts and those with cardiac abnormalities, using 2D STE and 4D e-STIC approach.

\section{Methods and Materials}

\section{Population}

This prospective study included fetuses between 20- and 40-weeks' gestation. Thirty-four control fetuses with accurate first or second trimester dating US exams were included. These fetuses were free of malformations or growth disturbances at the time of the inclusion and were referred to our clinic for second and third trimester ultrasound exams. Twenty-nine fetuses with congenital heart disease have been selected among fetuses evaluated in the fetal echocardiography clinic. The malformations were as follows: tetralogy of Fallot, ventricular septal defects, transposition of great arteries, dysplasia of tricuspid associated with pulmonary insufficiency, cardiomegaly with biventricular hypertrophy, aortic valve stenosis, aneurysm right atrium and cardiomyopathy of left ventricular with no-compacted myocardium.

Table 1a: fetal cardiac abnormalities split in 4 sub-groups: 1a (left heart disease), 1b (right heart disease), 1c (transposition of the great arteries), 1d (others). This division was used in the second phase of our study.

\begin{tabular}{|c|c|}
\hline $\begin{array}{c}\text { Fetal } \\
\text { cardiac abnormalities }(n=29)\end{array}$ & \\
\hline \multirow{9}{*}{$\begin{array}{c}\text { Group 1a: } \\
\text { Left heart disease } \\
\qquad(n=11)\end{array}$} & - Ventricular septal defect with Aortic valve dysplasia \\
\hline & - Ventricular septal defect $(n=3)$ \\
\hline & - Interrupted aortic arch with hypoplastic ascending aorta and multiple ventricular septal defects \\
\hline & - Ventricular septal defect with coarctation of aorta \\
\hline & - $\quad$ Multiple ventricular septal defects \\
\hline & - Inlet ventricular septal defects with multiple ventricular septal defects \\
\hline & - $\quad$ Aortic valve stenosis with bicuspid aortic valve \\
\hline & $\begin{array}{l}\text { - Severe left ventricular noncompaction with ventricular septal defect and tricuspid valve dyspla- } \\
\text { sia Ebstein-like }\end{array}$ \\
\hline & - $\quad$ Dilated left ventricle with ventricular septal defect \\
\hline \multirow{4}{*}{$\begin{array}{c}\text { Group 1b: } \\
\text { Right heart disease } \\
(n=5)\end{array}$} & - $\quad$ Tricuspid Valve Dysplasia (Non-Ebstein) with pulmonary atresia \\
\hline & - $\quad$ Tetralogy of Fallot with pulmonary valve stenosis \\
\hline & - $\quad$ Tetralogy of Fallot $(n=2)$ \\
\hline & - Double outlet right ventricle \\
\hline \multirow{3}{*}{$\begin{array}{l}\text { Group 1c: } \\
\text { Transposition of great arteries }(n=7)\end{array}$} & - $\quad$ Transposition of great arteries with ventricular septal defects $(n=3)$ \\
\hline & - $\quad$ Transposition of great arteries $(n=3)$ \\
\hline & - Transposition of great arteries with aneurysmatic patent foramen ovale \\
\hline \multirow{6}{*}{$\begin{array}{l}\text { Group 1d: } \\
\text { Others } \\
(n=6)\end{array}$} & - $\quad$ Atrioventricular block \\
\hline & - $\quad$ Cardiomegaly with biventricular hypertrophy and pericardial effusion \\
\hline & - Neoformation in right atrium \\
\hline & - Echogenic foci within the left ventricle \\
\hline & - $\quad$ Atrial flutter \\
\hline & - $\quad$ Aneurysmatic right atrium \\
\hline
\end{tabular}

Our study consisted of two phases: in the first one, fetuses have been divided in two groups (group 0: normal heart, group 1:congenital heart disease), while in the second phase fetuses affected by congenital heart disease have been split into 4 sub- groups (group 1a: left heart disease, group 1b: right heart disease, group 1c: transposition of the great arteries and group 1d: others) (Table 1a). 
All measurements on healthy and affected fetuses were performed by two sonographers experienced in fetal echocardiography (A.P., A.B.).

\section{Image acquisition and analysis}

Two-dimensional images of the 4-chambers were acquired using a RM6C or EM6C transducer of the Voluson E10 US system (GE Healthcare, Milwaukee, WI). Three second cine clips of the 4-chambers view were obtained and stored as Digital Imaging and Communications in Medicine files and exported to an offline database. E-STIC volumes were acquired using an electronic $4 \mathrm{D}$ probe, EM6C, using the option maximal quality. Once the 2D images and 4D volumes of the 4-chamber view were obtained and stored, they were examined using fetalHQ software (GE Healthcare; Zipf, Austria) using criteria that have been previously described $[5,15,18,20-22]$. Using the equation of Hadlock et al, estimated fetal weight (computing the measurements of the biparietal diameter, head circumference, abdominal circumference, and femur length) was expressed using z-score for each fetus [23,24]. At the end of the analysis, raw data were exported to an ASCII text file, later imported into an excel spreadsheet. In this study we focused on left ventricular global strain (GS), left ventricular ejection fraction (EF), left ventricular length and area in end-systole (ESL and ESA) and end-diastole (EDL and EDA), and left ventricular fractional area change (FAC).

\section{Ethics}

Each patient of the study signed a consent form at recruitment. The study protocol was approved by the local Ethics Committee of Sant' Orsola-Malpighi Hospital and a consent form signed at recruitment was obtained from each eligible patient (575/2018/ Oss/AOUBo). The study protocol conforms to the ethical guidelines of the "World Medical Association (WMA) Declaration of Helsinki-
Ethical Principles for Medical Research Involving Human Subjects" adopted by the 18th WMA General Assembly, Helsinki, Finland, June 1964 and amended by the 59th WMA General Assembly, Seoul, South Korea, October 2008.

\section{Statistics}

Categorical variables were expressed as percentage, while continuous variables were expressed as mean \pm standard deviation if they are normally distributed. Differences between categorical variables were analyzed by using Chi-Quadro Test, while continuous variables by one-way ANOVA Test. A p-value lesser than 0,05 was considered statistically significative. All the analyses were done through STATA/IC 15.1

\section{Result}

The study included 62 fetuses recruited between October 2018 and May 2019, 34 with normal heart and 29 affected by congenital heart disease. Mean gestational age was 29,68 weeks $\pm 4,99$ days and mean z-score estimated fetal weight was 0,24 $\pm 1,26$. 2D-images have been obtained in 62 fetuses, while the 4D images by STIC in 59 cases.

\section{First phase}

The population was homogeneously distributed in the mentioned groups. No differences were found between the groups in regard to gestational age (GA 29,34 $\pm 4,63$ vs 30,09 $\pm 5,44$ ) and estimated fetal weight (EFW $0,25 \pm 0,22$ vs $0,22 \pm 0,31$ ) at the time of examination (Table $1 \mathrm{~b}$ ). In Table 2 are summarized the measured values obtained with 2D and 4D e-STIC techniques. Compared to normal fetuses using 2D imaging, there was no statistically difference of EF, ESL, ESA, EDL, EDA, FAC, GS. There were not found any significantly different between the two groups of EF, ESL, ESA, EDL, EDA, FAC and GS obtained with 4D e-STIC.

Table 1b: gestational ages (expressed in weeks) and estimated fetal weight (expressed in z-score) of the two groups. Values are expressed as mean \pm SD. Group 0 includes fetuses with normal heart. Group 1 includes fetuses with congenital heart disease. (G DAYS gestational age in days; G WEEKS gestational age in weeks, EFW estimated fetal weight).

\begin{tabular}{|c|c|c|c|}
\hline \multirow{2}{*}{ G WEEKS } & GROUP & MEAN \pm SD & IC 95\% \\
\hline \multirow{2}{*}{ EFW z-score } & 0 & $29,34 \pm 4,63$ & $27,72-30,95$ \\
\cline { 2 - 4 } & 1 & $30,09 \pm 5,44$ & $28,02-32,17$ \\
\cline { 2 - 4 } & 1 & $0,25 \pm 0,22$ & $-0,20-0,70$ \\
\hline \multirow{2}{*}{0} & $0,22 \pm 0,31$ & $-0,45-0,90$ \\
\hline
\end{tabular}

Table 2: Summary table of the first phase of the study. 0: fetuses with normal heart. 1: fetuses affected by congenital heart disease. Values are expressed as mean \pm SD. EF: ejection fraction. ESL: end-systole left ventricular length. ESA: end-systole left ventricular area. EDL: end-diastole left ventricular length. EDA: end-diastole left ventricular area. FAC: fractional area change. GS: longitudinal global strain. STIC: images acquired using an electronic 4D probe. 2D: 2D images.

\begin{tabular}{|c|c|c|c|c|c|}
\hline & GROUP & NUMBER OF PATIENT & MEAN $\pm D S$ & ICC $95 \%$ & P-Value \\
\hline \multirow{2}{*}{ EF STIC } & 0 & 34 & $61,73 \pm 9,37$ & $58,4565,00$ & \multirow{2}{*}{0,762} \\
\hline & 1 & 25 & $60,70 \pm 16,55$ & $53,8667,53$ & \\
\hline \multirow{2}{*}{ EF 2D } & 0 & 34 & $59,58 \pm 9,78$ & $55,8662,69$ & \multirow{2}{*}{0,872} \\
\hline & 1 & 28 & $59,71 \pm 11,42$ & $55,2864,15$ & \\
\hline \multirow{2}{*}{ ESL STIC } & 0 & 34 & $1,60 \pm 0,37$ & $1,471,73$ & \multirow{2}{*}{0,272} \\
\hline & 1 & 25 & $1,48 \pm 0,41$ & $1,311,65$ & \\
\hline
\end{tabular}




\begin{tabular}{|c|c|c|c|c|c|}
\hline \multirow{2}{*}{ ESL 2D } & 0 & 34 & $1,57 \pm 0,34$ & $1,451,70$ & \multirow{2}{*}{0,537} \\
\hline & 1 & 28 & $1,63 \pm 0,37$ & $1,491,78$ & \\
\hline \multirow{2}{*}{ ESA STIC } & 0 & 34 & $0,97 \pm 0,43$ & $0,821,12$ & \multirow{2}{*}{0,431} \\
\hline & 1 & 25 & $0,88 \pm 0,40$ & $0,711,05$ & \\
\hline \multirow{2}{*}{ ESA 2D } & 0 & 34 & $0,91 \pm 0,38$ & $0,771,04$ & \multirow{2}{*}{0,361} \\
\hline & 1 & 28 & $1,01 \pm 0,45$ & $0,831,18$ & \\
\hline \multirow{2}{*}{ EDL STIC } & 0 & 34 & $1,99 \pm 0,39$ & $1,852,12$ & \multirow{2}{*}{0,589} \\
\hline & 1 & 25 & $1,92 \pm 0,47$ & $1,732,12$ & \\
\hline \multirow{2}{*}{ EDL 2D } & 0 & 34 & $1,93 \pm 0,36$ & $1,812,06$ & \multirow{2}{*}{0,144} \\
\hline & 1 & 28 & $2,09 \pm 0,48$ & $1,912,28$ & \\
\hline \multirow{2}{*}{ EDA STIC } & 0 & 34 & $1,75 \pm 0,62$ & $1,531.97$ & \multirow{2}{*}{0,768} \\
\hline & 1 & 25 & $1,70 \pm 0,73$ & $1,392,00$ & \\
\hline \multirow{2}{*}{ EDA 2D } & 0 & 34 & $1,60 \pm 0,57$ & $1,401,80$ & \multirow{2}{*}{0,169} \\
\hline & 1 & 28 & $1,84 \pm 0,77$ & $1,542,14$ & \\
\hline \multirow{2}{*}{ FAC STIC } & 0 & 34 & $46,13 \pm 8,51$ & $43,149,102$ & \multirow{2}{*}{0,529} \\
\hline & 1 & 25 & $47,62 \pm 9,60$ & $43,6651,59$ & \\
\hline \multirow{2}{*}{ FAC 2D } & 0 & 34 & $44,08 \pm 8,79$ & $41,0147,15$ & \multirow{2}{*}{0,723} \\
\hline & 1 & 28 & $44,89 \pm 8,96$ & $41,4148,36$ & \\
\hline \multirow{2}{*}{ GS\% STIC } & 0 & 34 & $-22,09 \pm 6,07$ & $-24,21-19,97$ & \multirow{2}{*}{0,173} \\
\hline & 1 & 25 & $-24,41 \pm 6,76$ & $-27,20-21,61$ & \\
\hline \multirow{2}{*}{ GS\% 2D } & 0 & 34 & $-20,19 \pm 6,74$ & $-22,55-17,84$ & \multirow{2}{*}{0,147} \\
\hline & 1 & 28 & $-22,87 \pm 7,58$ & $-25,81-19,93$ & \\
\hline
\end{tabular}

\section{Second phase}

We divided the group 1 in 4 subgroups: group 1a, left heart disease (11 patients), group $1 \mathrm{~b}$, right heart disease ( 5 patients), group 1c, transposition of the great arteries (7 patients) and group
$1 \mathrm{~d}$, others (6 patients with other cardiac diseases). Group 0 included 34 fetuses with normal heart. According to the value of gestational age (GA) and estimated fetal weight (EFW), the population was equally distributed in the mentioned groups (Table 3).

Table 3: gestational ages (expressed in weeks) and estimated fetal weight (expressed in z-score) of the five groups. $0=$ normal heart; $1 \mathrm{a}=$ left heart disease; $1 \mathrm{~b}=$ right heart disease; $1 \mathrm{c}=$ transposition of the great arteries; $1 \mathrm{~d}=$ =others.

\begin{tabular}{|c|c|c|c|c|c|c|}
\hline & 0 & $1 \mathrm{a}$ & $1 \mathrm{~b}$ & $1 \mathrm{c}$ & $1 \mathrm{~d}$ & $\mathrm{p}$-value \\
\hline GA WEEKS & $29,34 \pm 4,63$ & $27,74 \pm 5,62$ & $31,2 \pm 5,72$ & $31,20 \pm 5,88$ & $32,21 \pm 3,81$ & 0,352 \\
\hline EFW z-score & $0,25 \pm 1,30$ & $0,31 \pm 1,17$ & $-1,21 \pm 2,14$ & $0,36 \pm 0,21$ & $0,70 \pm 1,40$ & 0,541 \\
\hline
\end{tabular}

Table 4: Summary table of the second phase of the study. $0=$ normal heart; $1=$ left heart disease, 2 : right heart disease; $3=$ transposition of the great arteries; 4=others. EF: ejection fraction. ESL: end-systole left ventricular length. ESA: end-systole left ventricular area. EDL: end-diastole left ventricular length. EDA: end-diastole left ventricular area. FAC: fractional area change. GS: longitudinal global strain. STIC: images acquired using an electronic 4D probe. 2D: 2D images.

\begin{tabular}{|c|c|c|c|c|c|c|}
\hline Strain parameters & $\begin{array}{c}0 \\
\mathrm{~N}=34\end{array}$ & $\begin{array}{c}1 \\
N=9\end{array}$ & $\begin{array}{c}2 \\
N=5\end{array}$ & $\begin{array}{c}3 \\
N=6\end{array}$ & $\begin{array}{c}4 \\
N=5\end{array}$ & P-value \\
\hline ESL STIC & $1,60 \pm 0,37$ & $1,39 \pm 0,37$ & $1,39 \pm 0,38$ & $1,49 \pm 0,42$ & $1,75 \pm 0,51$ & 0,362 \\
\hline ESA STIC & $0,97 \pm 0,43$ & $0,77 \pm 0,27$ & $0,77 \pm 0,35$ & $0,82 \pm 0,36$ & $1,25 \pm 0,56$ & 0,232 \\
\hline EDL STIC & $1,99 \pm 0,39$ & $1,82 \pm 0,49$ & $1,79 \pm 0,44$ & $1,90 \pm 0,42$ & $2,29 \pm 0,50$ & 0,292 \\
\hline EDA STIC & $1,75 \pm 0,62$ & $1,49 \pm 0,54$ & $1,44 \pm 0,50$ & $1,61 \pm 0,72$ & $2,42 \pm 0,92$ & 0,09 \\
\hline FAC STIC & $46,13 \pm 8,51$ & $47,16 \pm 9,05$ & $46,47 \pm 7,78$ & $48,11 \pm 10,90$ & $49,04 \pm 13,23$ & 0,960 \\
\hline GS\% STIC & $-22,09 \pm 6,07$ & $-26,06 \pm 7,5$ & $-22,33 \pm 5,95$ & $-22,29 \pm 6,044$ & $-26,04 \pm 7,70$ & 0,420 \\
\hline EF STIC & $61,73 \pm 9,37$ & $57,67 \pm 13.42$ & $60,67 \pm 7,55$ & $62,89 \pm 12,89$ & $63,55 \pm 15,64$ & 0,913 \\
\hline Strain parameters & $\begin{array}{c}0 \\
\mathrm{~N}=34\end{array}$ & $\begin{array}{c}1 \\
N=11\end{array}$ & $\begin{array}{c}2 \\
N=5\end{array}$ & $\begin{array}{c}3 \\
\mathrm{~N}=6\end{array}$ & $\begin{array}{c}4 \\
\mathrm{~N}=6\end{array}$ & P-value \\
\hline ESL 2D & $1,57 \pm 0,34$ & $1,46 \pm 0,34$ & $1,57 \pm 0,30$ & $1,76 \pm 0,44$ & $1,88 \pm 0,30$ & 0,158 \\
\hline ESA 2D & $0,91 \pm 0,38$ & $0,87 \pm 0,38$ & $0,86 \pm 0,45$ & $1,09 \pm 0,54$ & $1,30 \pm 0,43$ & 0,200 \\
\hline
\end{tabular}




\begin{tabular}{|c|c|c|c|c|c|c|}
\hline EDL 2D & $1,93 \pm 0,36$ & $1,94 \pm 0,43$ & $1,91 \pm 0,59$ & $2,18 \pm 0,54$ & $2,44 \pm 0,30$ & 0,06 \\
\hline EDA 2D & $1,69 \pm 0,57$ & $1,67 \pm 0,73$ & $1,47 \pm 0,67$ & $1,97 \pm 0,97$ & $2,32 \pm 0,60$ & 0,117 \\
\hline FAC 2D & $44,08 \pm 8,79$ & $47,23 \pm 10,79$ & $40,59 \pm 11,52$ & $44,39 \pm 3,67$ & $44,67 \pm 7,08$ & 0,726 \\
\hline GS \% 2D & $-20,19 \pm 6,74$ & $-25,78 \pm 8,48$ & $-18,99 \pm 10,48$ & $-20,67 \pm 3,62$ & $-22,95 \pm 5,06$ & 0,204 \\
\hline EF 2D & $59,28 \pm 9,78$ & $62,45 \pm 13,50$ & $55,08 \pm 14,08$ & $59,53 \pm 6,97$ & $58,73 \pm 9,78$ & 0,782 \\
\hline
\end{tabular}

Table 4 summarizes the measurements obtained with both techniques. As in the first phase, the values of ESL, ESA, EDL, EDA, FAC, EF and GS acquired with 2D technique proved no significative differences between the five groups. Furthermore, there were not any statistically difference between five groups of ESL, ESA, EDL, EDA, FAC, EF and GS obtained with 4D e-STIC technique.

\section{Discussion}

This is the first study evaluating fetal left ventricular function by means of 2D and 4D e-STIC speckle-tracking echocardiography. We analyzed and compared several cardiac function parameters in normal fetal hearts and those with cardiac abnormalities. Speckle tracking is a semiautomated process, based on the tracking of 'speckles', conceptualized as small myocardial fingerprints, generated by ultrasound-myocardial tissue interactions during cardiac cycle. Specific algorithms allow to evaluate ventricular function [15]. Although 2D-speckle tracking is now considered equal or superior to Doppler techniques [12], thanks to its angle independency, it has several limitations $[6,25,26]$. The small size of the fetal heart, fetal movements and maternal breathing may affect the image resolution and the quality of the small tracking region. Trying to overcome its limits, recently, some authors experimented 3D approach to fetal speckle tracking echocardiography $[27,28]$. Our group had already outlined that 4D e-STIC technique can obtain optimal fetal heart volume in more than $90 \%$ of cases within the time frame of a standard examination of fetal anatomy [18,19,29].

Aiming to test these techniques to fetal heart abnormalities, we compared different parameters obtained by 2D and 4D e-STIC method in normal fetal hearts and those with cardiac defects. As we expected thanks to recent literature results, we did not find differences between the analyzed groups $[5,30]$ because congenital heart diseases included did not affect left ventricular systolic function. However, post-natal test on a fetus affected by severe left ventricular noncompaction showed a significative low longitudinal strain $(-13,9 \%)$ similarly to the value obtained during the fetal scan $(-15,05 \%)$. Different pathophysiology lie under different cardiac abnormalities. The heterogeneity and complexity of cardiac abnormalities can complicate speckle tracking analysis and its interpretation, because the size of ventricular chambers can differ between a cardiac heart defect and another. This technical problem can be added to the uncertainty about angle independency of STE. Furthermore, regarding the acquisition rate, the frame rate is dependent on the angle and depth used for the acquisition [15], but no standards have been established yet to ensure the speckles can be tracked throughout the cardiac cycle. In our study we used high frame rates in order to optimize the resolution.
STE is a well-known modality in pediatric and adult echocardiography to follow changes in ejection fraction in patients with cardiomyopathy and to understand the kind of ventricular disfunction [8]. Even if it is not a routine clinical test in fetal cardiology, STE has the potential to improve our understanding on fetal cardiac function. Firstly, it could lead to the early detection of cardiac disfunction in several clinical conditions, such as structural defects, cardiomyopathies and risky diseases like Parvovirus B19 infection [31]. Moreover, we believe that STE will be a useful tool to compute the prognosis of affected and supposedly affected fetuses, such as DeVore et al had recently proved in a cohort of 108 fetuses with suspected prenatal diagnosis of aortic coarctation [32]. Nevertheless, published studies make comparison between healthy and affected fetus difficult and the results are not homogeneous [8]. There is still a lack of normal values for fetal systolic and diastolic function through gestation [33]. Moreover, the heterogeneity and complexity of cardiac abnormalities can complicate speckle tracking analysis and its interpretation [8]. We believe that this study provides an interesting approach to fetal speckle tracking echocardiography introducing the innovative 4D e-STIC method for cardiac abnormalities research. In our cohort, 2D and 4D e-STIC imaging techniques have shown similar results for each parameter. We believe that this approach will make STE more feasible in the future.

\section{Conclusion}

The main limitation of this study is the requirement of specialized software and an electronic probe to simultaneously calculate cardiac function parameters using 2D and 4D e-STIC approach. The small number of CHD group might have limited the analysis. We need a larger cohort for a better understanding of the correlation between the analyzed parameters and fetal cardiac malformations. Moreover, we acknowledge that further studies on healthy fetuses and those with congenital heart defects will be required to test feasibility and reproducibility of this new imaging technique.

\section{Acknowledgement}

None.

\section{Conflict of Interest}

No conflict of interest.

\section{References}

1. Pacileo G, Di Salvo G, Limongelli G, Miele T, Calabrò R (2007) Echocardiography in congenital heart disease: usefulness, limits and new techniques. J Cardiovasc Med Hagerstown Md 8(1): 17-22. 
2. Godfrey ME, Messing B, Cohen SM, Valsky DV, Yagel S (2012) Functional assessment of the fetal heart: a review. Ultrasound Obstet Gynecol 39(2): 131-144.

3. van Dalen BM, Soliman OII, Vletter WB, Kauer F, van der Zwaan HB, et al. (2009) Feasibility and reproducibility of left ventricular rotation parameters measured by speckle tracking echocardiography. Eur J Echocardiogr 10(5): 669-676.

4. Cameli M, Mondillo S, Galderisi M, Mandoli GE, Ballo P, et al. (2017) Speckle tracking echocardiography: a practical guide. G Ital Cardiol 18(4): 253-269.

5. DeVore GR, Klas B, Satou G, Sklansky M (2017) Longitudinal Annular Systolic Displacement Compared to Global Strain in Normal Fetal Hearts and Those With Cardiac Abnormalities. J Ultrasound Medm 37(5): 11591171.

6. Geyer H, Caracciolo G, Abe H, Wilansky S, CarerjS, et al. (2010) Assessment of Myocardial Mechanics Using Speckle Tracking Echocardiography: Fundamentals and Clinical Applications. J Am Soc Echocardiogr 23(4): 351-369.

7. Goffinet C, Chenot F, Robert A, Pouleur A-C, de Waroux J-B le $\mathrm{P}$, Vancrayenest D, et al. (2009) Assessment of subendocardial vs. subepicardial left ventricular rotation and twist using two-dimensional speckle tracking echocardiography: comparison with tagged cardiac magnetic resonance. Eur Heart J 30(5): 608-617.

8. Day TG, Charakida M, Simpson JM (2019) Using speckle tracking echocardiography to assess fetal myocardial deformation: are we there yet. Ultrasound Obstet Gynecol 54(5): 575-581.

9. Saito K, Okura H, Watanabe N, Hayashida A, Obase K, et al. (2009) Comprehensive Evaluation of Left Ventricular Strain Using Speckle Tracking Echocardiography in Normal Adults: Comparison of ThreeDimensional and Two-Dimensional Approaches. J Am Soc Echocardiogr 22(9): 1025-1030.

10. Horton KD, Meece RW, Hill JC (2009) Assessment of the Right Ventricle by Echocardiography: A Primer for Cardiac Sonographers. J Am Soc Echocardiogr 22(7): 776-792.

11. Mondillo S, Galderisi M, Mele D, Cameli M, Lomoriello VS, et al. (2011) Speckle-Tracking Echocardiography. J Ultrasound Med 30(1): 71-83.

12. Sutherland GR, Di Salvo G, Claus P, D’hooge J, Bijnens B (2004) Strain and strain rate imaging: a new clinical approach to quantifying regional myocardial function. J Am Soc Echocardiogr 17(7): 788-802.

13. Di Salvo G, Russo MG, Castaldi B, Di Bello V, Antonini-Canterin F, et al. (2006) Evaluation of ventricular function in the fetus. G Ital Cardiol 10(8): 499-508.

14. Di Salvo G, Caso P, Pacileo G, Severino S, Miele T, et al. (2005) New echocardiographic techniques in the study of coronary artery disease: strain and strain rate. Ital Heart J Suppl Off J Ital Fed Cardiol 6(1): 17-24.

15. DeVore GR, Polanco B, Satou G, Sklansky M (2016) Two-Dimensional Speckle Tracking of the Fetal Heart. J Ultrasound Med 35(8): 1765-1781.

16. Ahmed BI (2014) The new 3D/4D based spatio-temporal imaging correlation (STIC) in fetal echocardiography: a promising tool for the future. J Matern Fetal Neonatal Med 27(11): 1163-1168.

17. DeVore GR, Falkensammer P, Sklansky MS, Platt LD (2003) Spatiotemporal image correlation (STIC): new technology for evaluation of the fetal heart. Ultrasound Obstet Gynecol 22(4): 380-387.

18. DeVore GR, Falkensammer P, Sklansky MS, Platt LD (2003) Spatiotemporal image correlation (STIC): new technology for evaluation of the fetal heart. Ultrasound Obstet Gynecol Off J Int Soc Ultrasound Obstet Gynecol 22(4): 380-387.
19. DeVore GR, Satou G, Sklansky M (2017) 4D fetal echocardiography-An update. Echocardiography 34(12): 1788-1798.

20. Bertini M, Mollema SA, Delgado V, Antoni ML, Ng ACT, et al. (2009) Impact of Time to Reperfusion After Acute Myocardial Infarction on Myocardial Damage Assessed by Left Ventricular Longitudinal Strain. Am J Cardiol 104(4): 480-485.

21. DeVore GR (2005) Assessing fetal cardiac ventricular function. Semin Fetal Neonatal Med 10(6): 515-541.

22. DeVore GR, Klas B, Satou G, Sklansky M (2019) Quantitative evaluation of fetal right and left ventricular fractional area change using speckletracking technology. Ultrasound Obstet Gynecol 53(2): 219-228.

23. Hadlock FP, Deter RL, Harrist RB, Park SK (1984) Estimating fetal age: computer-assisted analysis of multiple fetal growth parameters. Radiology 152(2): 497-501.

24. Hadlock FP, Harrist RB, Sharman RS, Deter RL, Park SK (1985) Estimation of fetal weight with the use of head, body, and femur measurements--a prospective study. Am J Obstet Gynecol 151(3): 333-337.

25. Langeland S, D'hooge J, Claessens T, Claus P, Verdonck P, et al. (2004) RF-based two-dimensional cardiac strain estimation: a validation study in a tissue-mimicking phantom. IEEE Trans Ultrason Ferroelectr Freq Control 51(11): 1537-1546

26. Langeland S, D'hooge J, Wouters PF, Leather HA, Claus P, et al. (2005) Experimental Validation of a New Ultrasound Method for the Simultaneous Assessment of Radial and Longitudinal Myocardial Deformation Independent of Insonation Angle. Circulation 112(14): 2157-2162.

27. Enzensberger C, Achterberg F, Graupner 0, Wolter A, Herrmann J, et al. (2017) Wall-motion tracking in fetal echocardiography-Influence of frame rate on longitudinal strain analysis assessed by two-dimensional speckle tracking. Echocardiography 34(6): 898-905.

28. Nemes A, Katona M, Kalapos A, Domsik P, Forster T (2014) Threedimensional speckle tracking echocardiographic analysis of a fetal heart with hypoplastic left heart syndrome--a case from the MAGYAR-Fetus Study. Int J Cardiol 176(3): e81-82.

29. Guasina F, Bellussi F, Morganelli G, Salsi G, Pilu G, et al. (2018) Electronic spatiotemporal image correlation improves four-dimensional fetal echocardiography. Ultrasound Obstet Gynecol 51(3): 357-360.

30. Germanakis I, Matsui H, Gardiner HM (2012) Myocardial Strain Abnormalities in Fetal Congenital Heart Disease Assessed by Speckle Tracking Echocardiography. Fetal Diagn Ther 32(1-2): 123-130.

31. Ornoy A, Ergaz Z (2017) Parvovirus B19 infection during pregnancy and risks to the fetus. Birth Defects Res 109(5): 311-323.

32. DeVore GR, Haxel C, Satou G, Sklansky M, Pelka MJ, et al. (2020) Improved detection of coarctation of the aorta using speckle tracking analysis of the fetal heart using the last examination prior to delivery. Ultrasound Obstet Gynecol.

33. Maskatia SA, Pignatelli RH, Ayres NA, Altman CA, Sangi-Haghpeykar H, et al. (2016) Longitudinal Changes and Interobserver Variability of Systolic Myocardial Deformation Values in a Prospective Cohort of Healthy Fetuses across Gestation and after Delivery. J Am Soc Echocardiogr Off Publ Am Soc Echocardiogr 29(4): 341-349. 